\title{
Emotional and Behavioral Difficulties in Adolescents during COVID- 19: Implications for Planning Community Based Interventions
}

\author{
Sangeeta Bhatia', $\underline{\text { Sanjana Seth }}^{2}$ \\ ${ }^{1}$ Associate Professor, Department of Psychology, Gargi College, University of Delhi, New Delhi, India. \\ ${ }^{2}$ Psychologist \& Psychotherapist, Chandimandir Command Hospital, Chandigarh, India. \\ DOI: https://doi.org/10.24321/2349.2880.202015
}

\section{I $\quad \mathbf{N} \quad \mathbf{F} \quad \mathbf{O}$}

\section{Corresponding Author:}

Sangeeta Bhatia, Department of Psychology, Gargi College, University of Delhi, New Delhi, India.

E-mail Id:

sangeeta.bhatia@gargi.du.ac.in

Orcid Id:

https://orcid.org/0000-0003-3650-1125

How to cite this article:

Bhatia S, Seth S. Emotional and Behavioral Difficulties in Adolescents during COVID-19: Implications for Planning Community Based Interventions. Ind J Youth Adol Health 2020; 7(4): 1-7.

Date of Submission: 2021-02-09

Date of Acceptance: 2021-03-09

\section{$\begin{array}{llllllll}\mathbf{A} & \mathbf{B} & \mathbf{S} & \mathbf{T} & \mathbf{R} & \mathbf{A} & \mathbf{C} & \mathbf{T}\end{array}$}

Background: The study aimed at assessing the emotional and behavioral difficulties in adolescents using the Strengths and Difficulties questionnaire. The challenges brought forth due to the COVID-19 shut down of educational institutions has created additional distress due to a lack of routine and separation from one's peer group. Being closeted with a family for prolonged duration can exacerbate pre-existing vulnerabilities of conflict and mental health issues.

Method: Both males and females were selected for the study. The age of the participants was $16-18$ years. The total sample size was 140 (mean age 17.5, SD 2.5), with 70 males (mean age- 17, SD 1.5) and 70 females (mean age - 16.5, SD 2.8). The Strengths and Difficulties Questionnaire by Goodman et al. (1998) was used, as also a detailed clinical case study was taken to identify pertinent issues and concerns.

Result: The study found significant gender differences in prosocial behavior and peer relationships in adolescents, and we assume that a role parity and empathy may be the linking factor between prosocial behavior and peer relationships. A case study is also discussed from the practitioner's perspective that brings into focus the increased consultations due to the stressful times. Findings also bring out a need for inculcating life skills in adolescents for recognizing and managing their stressors; incorporating a balance between internalizing and sharing their feelings. Conclusion: Interventions such as yoga, physical activities that maintain distance such as aerobics to mediate stress under lockdown can be undertaken at community level. The study showed that correlations may be present between conduct problems and emotional behaviors due to similar causal factors but more research is needed in the said field.

Keywords: Adolescence, Strengths, Difficulties, Emotional Regulation, Interventions 


\section{Introduction}

Adolescence marks the physical, psychological, and emotional transition of children into adulthood. Jodi A. Quad writes that the transition from being a child, dependent upon one's parents, to an independent and self-reliant adult, that is, the adolescent transition, represents one of the most dynamic, broad and influential periods of human development. The changes that occur during this period are sweeping, spanning biological, physical, psychological and behavioral domains of functioning. ${ }^{1}$

The World Health Organization focuses on changes in adolescence on the following three areas: Physical Changes - rapid biological changes witnessed by children. The growth spurt is a hallmark of these changes. Neurodevelopmental Changes - neuronal changes take place in both regions of the brain, predominantly the limbic system, as well as at the level of Pre-Frontal Cortex (PFC). These changes are responsible for pleasure seeking and reward processing, emotional responses and sleep regulation. And the PFC enables the executive functions: decision-making, organization, impulse control and planning for the future and, psychological and social changes. The psychosocial and emotional changes and increasing cognitive and intellectual capacities now determine the enhanced needs of the adolescents for peer support as well as identity development. The expression of which which differ among cultures and societies, based on social values and norms and the changing roles, responsibilities, relationships and expectations of this period of life. ${ }^{2}$

These rapid changes have implications for the mental wellbeing of the adolescent. Even before the COVID-19 pandemic caused challenges, mental illness has shown an upward trend in this age group. Anxiety, loneliness, anxiety, sleep disturbances have been a hallmark of disturbances in daily routines of this age group. Suicide rates are increasing and the need to provide access and better mental health support has been a priority of policy makers. These problems are likely to be aggravated due to unprecedented interruptions to the adolescents' daily lives and it is anticipated that these disruptions may precipitate further incidence of mental illness. Adolescents thrive on peer connections and social engagement. With educational institutions closing down, physical distancing being mandatory for safety, staying at home became mandatory. This disconnect with friends though a possibility through the online medium would not be a luxury for the economically disadvantaged. Preexisting conditions would likely be exacerbated and put the adolescent on a downward spiral of poorer mental health. It is likely that households with youngsters would see greater irritability, inattention, loss of appetite, disturbed sleep as further manifestations of the lockdown. This is due to lack of structure and routine to the day that is typically provided by a school schedule. Further, the curtailment of travel has implications for higher education and the admissions, delayed examination, absence of internship or opportunities for a career beginning. The ubiquitous use of internet otherwise as a dominant feature of one's life now becomes the only escape route from a confined and mundane space that feels restrictive and increasing anxiety because of the uncertainty to its end.

Erik Erikson, describes this stage for children that is important for the developmental task of identity vs. role confusion. Adolescents struggle with questions such as "Who am I?" and "What do I want to do with my life?" Those adolescents who develop a strong sense of identity are also able to withstand a challenge to their existing beliefs in comparison to those who fail to establish a stable identity. The latter experience challenges in their adult role as well that is exhibited through poor decision making and choices that have negative consequences for their well-being. ${ }^{3}$ The social isolation brought on by the pandemic has taken away this very rudimentary context for socio-emotional development and is expected to further fuel the grief and create anxieties due to a loss of sense of relational self.

Research has validated the issues in adolescence. ${ }^{4}$ In the years following puberty, adolescents experience both separation and individuation, and the implication is that the challenges of staying enmeshed with family for prolonged durations in the lockdown may be stressful. ${ }^{5} \mathrm{At}$ the same time, rates of risky behavior (e.g., substance use, delinquency, sexual activity) also rise markedly aggravated by the anxiety, conflict or distress, especially among boys. ${ }^{6}$ Adolescence development is an exchange of an intersection of biological, emotional, social and institutional processes. The development of problem behavior in early adolescence can be predicted from the absence of stable contexts such as family. ${ }^{7}$ Parents are the first to notice a marked change in early adolescents through their mood changes and emotional outbursts. ${ }^{8}$ These rollercoaster expression of emotions are likely to manifest more frequently when lack of routine in COVID times does not allow a balance with more appealing activities such as time spent with friends, or socializing or attending classes. The social world is all there is that gives a salient meaning to the adolescent's daily life. The understanding from the vast available research is that adolescent development happens in the social context, and every social experience helps integrate the identity into a more cohesive one through which the adolescent now views the world. ${ }^{5}$ Research has investigated gender differences in the relationship between sources of perceived support (parent, teacher, classmate, friend, school) and psychological and academic adjustment. Findings showed associations between all sources of support with depressive symptoms, anxiety, self-esteem, and academic adjustment. Parental support 
was a robust predictor of adjustment for both boys and girls and classmates' support was a unique predictor for boys. These results clearly demonstrate the significance of peer support in the social experience of adolescents. ${ }^{9}$ The findings indicate implications for increased vulnerabilities of adolescents due to loss of social contact with their peers in the pandemic times.

Turning to mental health, adolescence also marks the emergence of gender differences in depression with incidence showing greater number of females in this category. ${ }^{10}$ The psychosocial factors contributing to depression in females has been attributed to poor selfregulation of daily stressors, unrealistic expectations of societal standards for a certain way of physical appearance, negative experience of pubertal changes, and having a poor self-concept. ${ }^{11}$

Socioeconomic status remains a significant variable in determining mental health outcomes for children. ${ }^{12}$ The behavioral/ emotional problems in pre-school children can continue to persist up to adolescence. ${ }^{13}$ In 2015-16, the National Mental Health Survey of India conducted a nation-wide survey to assess the prevalence of a spectrum of mental health problems across the country. In the survey, it was found that the prevalence of mental disorders in age group 13-17 years was $7.3 \%$ and was nearly equal in both genders. Surveys in India show that "nearly 9.8 million of young Indians aged between 13-17 years would benefit from interventions. Prevalence of mental disorders was nearly twice (13.5\%) as much in urban metros as compared to rural (6.9\%) areas." Both familial and societal factors interfere in their growth, development, education and day to day social interactions. ${ }^{14,15}$ These researches highlight the pre-existing incidences of mental health issues and it can be safely said that with the current and prolonged social isolation, as well as economic deprivation, these statistics are likely to show an upward spiral.

In order to help adolescents grow independently and healthily, a number of intervention strategies can be implemented. Various studies have discussed the merits and de-merits of plethora of intervention strategies. ${ }^{16}$ The familial dynamics find salience in reducing negative behaviors such as conduct problems and emotional difficulties, whereas improving peer attachment and school connectedness could be important for the display of prosocial behavior, ${ }^{17}$ however these are time consuming and though needed, are a challenge in times of physical distancing. Stormshak writes that family centric approaches can be very helpful intervention strategies for adolescents. They define and govern their daily life and influence family decision making that improved behavioral and health outcomes for children. ${ }^{18}$ Therefore, family support groups can be the first line of intervention modules.
In adolescence, youth spend a high proportion of their time with their peers and in school; it is hardly surprising therefore that perceptions of peer and school norms have a strong influence on their attitudes and behaviors. A study on youth in Norway found that positive school norms were associated with increased prosocial behavior, but only though better quality contact. The results have implications for school-based interventions that aim to improve intergroup relations and highlight the importance of peer networks to promote more positive outgroup behaviors. ${ }^{19}$ Schools can thus identify measures that use the online medium while educational institutions stay shut to provide opportunities for the students to interact with their peers beyond formal classroom teaching.

Yoga is an important intervention strategy in increasing the social, mental, and physical well being of individuals. One study discusses yoga as a potential tool for adolescents to deal with stress and regulate themselves and therefore enhance their mental health. Here, since yoga in schools helps students improve resilience, mood, and self-regulation skills pertaining to emotions and stress, ${ }^{20}$ this idea can be implemented through neighborhood groups initiating yoga for adolescents during school closures in alleviating distress.

Research on mindfulness meditation for training teachers at two schools in the outer suburbs of Melbourne, Australia was initiated and the findings showed that there was a significant decrease in overall average score and the number of children in the diagnostic categories, $25.6 \%$ scoring in the borderline or diagnostic category for the SDQ pre-program and $16.3 \%$ post-program. ${ }^{21,22}$ Community-based creative activities had some positive effect on behavioral changes, self-confidence, self-esteem, levels of knowledge, and physical activity. Evidence from digital platforms supported Internet-based prevention and treatment programs for anxiety and depression; Exercising and increased physical activity was found to be effective in improving selfesteem and reducing depression score with no impact on anxiety scores. Psychological therapy when compared to antidepressants was found to have comparable effect on remission, dropouts, and depression symptoms. ${ }^{22}$

The present study was designed with the objective to determine the Strengths and Difficulties of the adolescent during the present challenging times. Review of research described here has unequivocally indicated the existing mental health concerns in this age group and the interventions that have been found to be useful. By identifying the concerns of adolescents it is hoped that interventions would be implemented as a priority. With this objective, a standardized measure the Strengths and Difficulties Questionnaire, and a case study from the practitioner's clinic is a integrated for a more holistic discussion. 


\section{Method}

Participants: Both males and females were selected for the study. The age of the participants was $16-18$ years. For the study, purposive sampling was chosen, since the participants were from a particular age and geographical region. The total sample size was 140 (mean age 17.5, SD $2.5)$, with 70 males (mean age- 17, SD 1.5) and 70 females (mean age- 16.5 , SD 2.8).

Measure: The Strengths and Difficulties Questionnaire23 was distributed amongst participants after their consent was taken, and analyzed on each dimension to study if there were differences on these between the males and females, as also on their total profile on the difficulties. The 25 item behavioral screening questionnaire has a three point rating (not true, somewhat true, certainly true). It has four problem scales; emotional symptoms (D1), conduct problems (D2), hyperactivity/ inattention (D3), peer relationship problems (D4), each scale's range of scores being $0-10$, which add to generate a total difficulties score (0-40). A fifth scale assesses positive aspects of prosocial behavior (D5). The internal reliability of the various selfreport scales is reported using Cronbach's alpha coefficient. This was 0.82 for the total difficulties, 0.75 for emotional symptoms, 0.72 for conduct problems, 0.69 for hyperactivity, 0.65 for prosocial behavior, and 0.61 for peer problems. ${ }^{23}$

Scoring-For scoring, the items marked as 'not true' received a score of 0 , the items marked as 'somewhat true' received the score of 1 , and the items marked as 'certainly true' received the score of 2 . However, for certain items, 7,11,14,21,25 reverse scoring was used. This was because these items, as opposed to the other items, had negative connotations, where 'not true' would have meant behaviors that need to be looked into. Therefore, for these items, 'not true' was marked as a 2, and 'certainly true' was marked by a 0 .

As per the Manual: "The original British cut-off points were derived by classifying approximately $10 \%$ of the normative sample with the most extreme scores in the "abnormal" banding, the next $10 \%$ in the "borderline" banding and the remaining $80 \%$ in the "normal" banding categories." Some studies in Europe, Asia and Africa have used scores above the 90th percentile of their samples to define the 'abnormal' group. ${ }^{24}$ However, owing to different norms and cultural trends in India, the cut off points were derived from the study done on college students in Bangalore in 2016.

Table I.Cut off Scores

\begin{tabular}{|c|c|c|}
\hline SDQ Domains & Males & Females \\
\hline Total Difficulties & 22 & 23 \\
\hline Emotional symptoms & 8 & 9 \\
\hline Conduct Problmes & 6 & 5 \\
\hline Hyperactivity & 7 & 7 \\
\hline Peer problems & 6 & 6 \\
\hline Prosocial behaviour & 4 & 5 \\
\hline
\end{tabular}

Table 2.Mean and Standard Deviation with respect to gender on the 5 Domains and Total Difficulties

\begin{tabular}{|c|c|c|c|c|c|c|}
\hline Domains & Males (n 70) & S.D. & Females (n 70) & S.D. & Standard error of difference & $\mathbf{t}(\mathbf{1 3 8 )}$ \\
\hline D1 & 3.5 & 2.3 & 3.8 & 2.2 & .38 & 0.78 \\
\hline D2 & 3.2 & 1.6 & 3.3 & 1.6 & 0.27 & 0.36 \\
\hline D3 & 2.8 & 1.7 & 2.6 & 1.7 & .28 & .69 \\
\hline D4 & 4.3 & 2.0 & 3.5 & 1.8 & .32 & $2.48^{*}$ \\
\hline D5 & 7.6 & 1.6 & 8.3 & 1.8 & .28 & $2.43^{*}$ \\
\hline Total Difficulties & 14.5 & 5.4 & 13.7 & 5.2 & 0.89 & 0.89 \\
\hline${ }^{*}<$.05
\end{tabular}

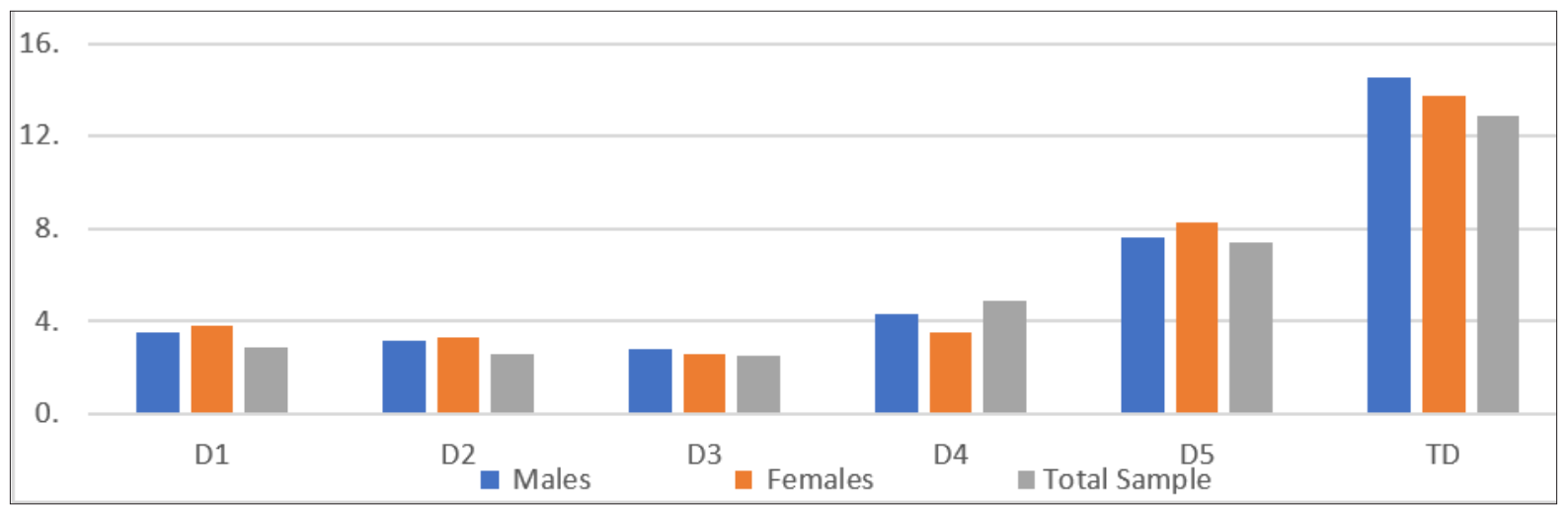

Figure I.Bar Graph Depicting the Means on the Five Domains for Males and Females 


\section{Result}

The present study aims to assess the emotional and behavioral difficulties in adolescents using the Strengths and Difficulties Questionnaire..$^{23}$ The Dimensions studied by the SDQ are- Emotional Behavior, Conduct Problems, Hyperactivity, peer problems, and prosocial behavior, first four dimensions are added to reveal the total difficulties. While the first four domains are negative, they assess traits or the 'problems' being faced by the adolescents, while the fifth domain is a positive one, and assesses the positive traits or characteristics (Table 1). This study becomes all the more pertinent in the present scenario of pandemic where work from home and online schooling are the new norm. Family members have been cloistered together due to the restrictive strategies put in place by the State to tackle the pandemic. Paucity of space, lack of privacy, extended family circles etc have started taking a toll on the well-being of adolescents as well as adults.

In the total compilation, it was found that the mean of the total sample was 14.1 in Total difficulties, 3.7 in emotional behavior, 3.4 in conduct problem, 2.8 in hyperactivity, 3.9 in peer relationships, and 8.0 in prosocial behavior. The Standard deviation for the domains was 5.2, 2.4, 2.8, 1.9, 1.9 , and 1.8 respectively.

The typical female profile showed that the mean of the total difficulties was 13.7, for emotional behavior it was 3.8, for conduct problems it was 3.3, for hyperactivity it was 2.6, 3.5 for peer relationships and 8.3 for prosocial behavior. Thus, the highest value was depicted by emotional behavior, whereas and prosocial behavior was higher than that in the cut offs. This indicates that the females in the study may have slight emotional problems, as compared to other domains, however, they do not exceed the cut off limit, indicating that they aren't causing any mal-adaptiveness. One of the reasons for females to show higher on emotional problems in this study may be due to a general tendency to internalize their feelings as compared to the adolescent boys. It is offset by a The higher prosocial behavior value which is a positive indicator, showing that the females in the study were more prosocial than the normative data. This trend is seen in healthcare settings wherein young girls present with anxiety or depressiveness affecting their academic performance visibly but social interaction (with peers) remains reasonably sustained (Table 2 ).

The typical male profile showed that the mean of the total difficulties was 14.5 , for emotional behavior it was 3.5 , for conduct problems it was 3.2 , for hyperactivity it was $2.8,4.3$ for peer relationships, and 7.6 for prosocial behavior. This profile indicates that a male in the study may experience issues in peer relationships, as compared to others, since the mean for the same is slightly higher for other domains, however, it doesn't exceed the cutoff list. The data also indicates a higher value of prosocial behavior, which is an indicative of a positive trait, and can help in adaptiveness (Figure 1). In adolescent boys, the peer pressure frequently leans towards risk taking and experimenting which can further lead to unhealthy emotional behavior. Usually it gets reflected as oppositional defiance tendencies, aggression (targeted at self or adult family member).

It may be noted that no domain exceeds the cut offs given in scoring. However, the sample might include individuals who do exceed the cut off score, but the outlier was not reflected in the mean, but can be reflected in the standard deviation.

\section{Discussion}

Each domain is discussed separately for the two groups and a few important intervention techniques are henceforth discussed.

The peer relationships in the total sample not only indicated a higher mean, but also showed a significant disparity in the mean values of males and females. This is indicative of how males in particular might face difficulties in forming peer relationships during adolescence, as compared to females. A study found that self esteem plays a huge role in forming peer relationships and those adolescents with higher self esteem find it easier to form healthier peer relationships who have a higher self-esteem..$^{25}$ However, this does not count for the gender differences indicated in the study. Thus, the question thus emerges- are adolescent girls more empathetic and have a higher self-esteem than males? A study by Landazabal ${ }^{26}$ showed that girls showed greater empathy and were higher on prosocial behavior in contrast to boys who show higher profiles on the dimensions of aggression and antisocial behavior. This can also be corroborated by the fact that in the present study, girls scored higher than boys in prosocial behavior significantly. Graff and Carlo conducted a longitudinal study where study investigated the development of prosocial behavior across adolescence, and examined longitudinal associations with perspective taking and empathic concern. The results revealed marked gender differences in the development of prosocial behavior. Empathetic concern was consistently related to subsequent prosocial behavior. However, perspective taking was only indirectly related to prosocial behavior, via its effect on empathic concern. This indicates that the earlier the prosocial behavior starts more likely is the growth of empathic behaviors, which holds true only for females. ${ }^{27}$ It can be assumed that males would more likely feel challenged during the physical distancing in the pandemic times and find it difficult to express empathy to family members. Sharing household work may be one way of supportive behavior.

Emotional difficulty also had a higher mean than the rest, 
though it did not exceed the cut off value. A study by Ferdinand revealed that high rates of withdrawal from social contacts, anxiety or depression, somatic complaints without known medical origin, social problems, attention problems, delinquent behavior, and aggressive behavior during adolescence were risk factors for specific types of psychopathology and maladjustment in adolescents. 6 In the current scenario of COVID-19, there is a considerable intake of adolescents in Out-Patient Department (OPD) of psychiatry in hospitals. To share a case briefly: 15 year old $M$ was brought to the OPD with complaints of irregular sleep hours, poor appetite, irritability and lack of interest in studies. All this was noticed by the parents during the lockdown and it had increased to the extent where the basic functioning of the youngster started getting impacted negatively. The case history showed no developmental delays or trauma; however a rigid (authoritative) parenting style was noticed. There was also sibling comparison; unrealistic expectations (parental) and sporadic incidents of harsh corporal punishment meted out by the father. Continuing with the case intake, the youngster brought out the peer pressure faced by him viz a viz hosting parties at upscale eateries which was beyond his family's economic provision. As the relationship/ rapport with his parents was not too healthy, $\mathrm{M}$ had been internalizing his frustrations since a considerable time and the enforced interactions with his family in closed confines over this prolonged period of pandemic led to $\mathrm{M}$ reaching a breaking point. The negative behavior of oppositional defiance had become predominant. Similarly, in another case of A (16 years old female), the recent changes in environment exacerbated the anxiety leading to panic \& ideation of self harm. One of the triggers was poor connectivity of mobile \& internet services leading to feelings of alienation from friends. As the youngster was in a boarding school but had to move in with her parents due to school closure, the interpersonal conflicts started getting flared up to the extent wherein A had to be brought in for psychological intervention.

Adjustment problems have been found to be correlated with emotional and conduct problems. ${ }^{28}$ Parenting styles have been found to determine directly or indirectly behaviors such as aggression or delinquency through the pathway of low self-esteem and higher levels of stress. ${ }^{29}$ Since the study did not show a significant mean of emotional difficulty, it did show similar means for emotional behaviors and conduct problems. For instance, in the individual data, P7 does show a high score in emotional behavior, thus, intervention for above mentioned causes may be necessary especially given the extenuating circumstances of living under COVID-19. Some intervention techniques have been mentioned in the introduction, including parental support, yoga, meditation, and online therapy sessions.

\section{Conclusion}

The study aimed at assessing the emotional and behavioral difficulties in adolescents experienced during the COVID-19 time. It found significant gender differences in prosocial behavior and peer relationships in adolescents, and we assume that a role parity and empathy may be the linking factor between prosocial behavior and peer relationships. This study also brings out a need for inculcating life skills in adolescents for recognizing and managing their stressors; incorporating a balance between internalizing and sharing their feelings. Interventions such as yoga, physical activities that maintain distance such as aerobics to mediate stress under lockdown can be undertaken at community level. Online peer support groups should be encouraged by educational institutions. The study also showed that correlations may be present between conduct problems and emotional behaviors due to similar causal factors but more research is needed in the said field.

\section{Conflict of Interest: None}

\section{References}

1. Quad J . Adolescence: A unique period of challenge and opportunity for positive development 2014 Retrieved 4 April 2020, from: https://www.apa.org/pi/ families/resources/newsletter/2014/12/adolescencedevelopment.

2. World Health Organization. Adolescent development 2020. Retrieved 4 April 2020, from https://www.who. int/maternal_child_adolescent/topics/adolescence/ development/en/.

3. Erikson, E: Childhood \& Society. 2005, Norton Publishers.

4. Compas B, Orosal P, Grant, K. Adolescent stress and coping: implications for psychopathology during adolescence. Journal of Adolescence 1993; 16(3): 331349. From: https://doi.org/10.1006/jado.1993.1028

5. Kroger J. Identity development: Adolescence through adulthood 2007. Sage Publications.

6. Ferdinand R, Verhulst F. Psychopathology from adolescence into young adulthood: an 8-year followup study. American Journal of Psychiatry 1995; 152(11): 1586-1594. From: https://doi.org/10.1176/ ajp.152.11.1586

7. González M, Casas F, Coenders G. A Complexity Approach to Psychological Well-Being in Adolescence: Major Strengths and Methodological Issues. Social Indicators Research 2007; 80(2): 267-295. From: https://doi.org/10.1007/s11205-005-5073-y

8. Laitinen-Krispijn S, Van der Ende J, HazebroekKampschreur A et al. Pubertal maturation and the development of behavioural and emotional 
problems in early adolescence. Acta Psychiatrica Scandinavica 1999; 99(1): 16-25.From: https://doi. org/10.1111/j.1600-0447.1999.tb05380.x

9. Rueger S, Malecki C, Demaray M. Relationship Between Multiple Sources of Perceived Social Support and Psychological and Academic Adjustment in Early Adolescence: Comparisons Across Gender. Journal Of Youth And Adolescence 2010; 39(1): 47-61. From: https://doi.org/10.1007/s10964-008-9368-6

10. Hankin B, Mermelstein R, Roesch L. Sex differences in adolescent depression: Stress exposure and reactivity models. Child Dev 2007; 78: 279-295.

11. Crosnoe R, Johnson M. Research on Adolescence in the Twenty-First Century. Annual Review of Sociology 2011; 37(1): 439-460. From: https://doi.org/10.1146/ annurev-soc-081309-150008

12. Goldstein E, Gonzales G, Meara E. How do economic downturns affect the mental health of children? Evidence from the National Health Interview Survey. Health Economics 2019; 28(8): 955-970. From: https://doi.org/10.1002/hec.3885

13. Gutgesell M, Payne N. Issues of Adolescent Psychological Development in the $21^{\text {st }}$ Century. Pediatrics in Review 2004; 25(3): 79-85. Form: https://doi.org/10.1542/ pir.25-3-79

14. National Mental Health Survey of India (NHMS): Summary of Survey, 2016 NIHMHANS.ac.in

15. Holmbeck G, Leake C. Separation-Individuation and Psychological Adjustment in Late Adolescence. Journal Of Youth And Adolescence 1999; 28(5): 563-581. https:// doi.org/10.1023/a:1021654626328

16. Gleason K, Jensen-Campbell L, Ickes W. The Role of Empathic Accuracy in Adolescents' Peer Relations and Adjustment. Personality and Social Psychology Bulletin 2009; 35(8): 997-1011. Retrieved https://doi. org/10.1177/0146167209336605

17. Oldfield J, Humphrey N, Hebron J. The role of parental and peer attachment relationships and school connectedness in predicting adolescent mental health outcomes. Child nd Adolescent Mental Health 2016; 21(1): 21-29. From: https://doi.org/10.1111/ camh.12108

18. Stormshak EA, Chronister KM, Caruthers AS. The impact of Family-Centred Prevention on Self-Regulation and Subsequent Long-Term Risk in Emerging Adults. Prevention Science 2017; 19(4): DOI: 10.1007/s11121017-0852-7

19. McKeown S, Taylor L. Perceived peer and school norm effects on youth antisocial and prosocial behaviours through intergroup contact in Northern Ireland. British Journal Of Social Psychology 2018; 57(3): 652-665. https://doi.org/10.1111/bjso.12257

20. Hagen I, Nayar U. Yoga for Children and Young People's
Mental Health and Well-Being: Research Review and Reflections on the Mental Health Potentials of Yoga. Frontiers In Psychiatry 2014; 5. From: https:// doi.org/10.3389/fpsyt.2014.00035

21. Joyce A, Etty-Leal, J, Zazryn T et al. A: Exploring a Mindfulness Meditation Program on the Mental Health of Upper Primary Children: A Pilot Study. Advances In School Mental Health Promotion 2010; 3(2): 17-25. https://doi.org/10.1080/1754730x.2010.9715677

22. Das JK, Salam RA, Lassi Z. A: Interventions for Adolescent Mental Health: An Overview of systematic reviews. Journal of Adolescent Health 2016; 59(4S): S49-S60. Retrieved online doi: 10.1016/j.jadohealth.

23. Goodman R, Meltzer H, Bailey V. The strengths and difficulties questionnaire: A pilot study on the validity of the self-report version. European Child \& Adolescent Psychiatry 1998; 7(3): 125-130. Form: https://doi. org/10.1007/s007870050057

24. Bhola P, Sathyanarayanan V, Rekha D et al. Assessment of self-reported emotional and behavioral difficulties among pre-university college students in Bangalore, India. Indian Journal of Community Medicine. 2016; 41(2): 146. Form: https://doi.org/10.4103/09700218.177536

25. Bosacki B, Dane A, Marini Z. Peer relationships and internalizing problems in adolescents: mediating role of self-esteem. Emotional And Behavioural Difficulties 2007; 12(4): 261-282. Retrieved online: https://doi. org/10.1080/13632750701664293

26. Landazabal M. A comparative analysis of empathy in childhood and adolescence: Gender differences and associated socio-emotional variables. International Journal of Psychology and Psychological Therapy 2009; 9(2): 217-235. Retrieved 4 April 2020, from.

27. Graaf JV, Carlo G, Crosetti E. Prosocial Behavior in Adolescence: Gender Differences in Development and Links with Empathy. Journal of Youth Adolescence 2017; 47(5): 1086-1099.

28. Achenbach T, McConaughy S, Howell C. Child/adolescent behavioral and emotional problems: Implications of cross-informant correlations for situational specificity. Psychological Bulletin 1987; 101(2): 213-232. Form: https://doi.org/10.1037/0033-2909.101.2.213

29. Finkenauer C, Engels R, Baumeister R. Parenting behavior and adolescent behavioural and emotional problems: The role of self-control. International Journal of Behavioral Development 2005; 29(1): 58-69. From: https://doi.org/10.1080/01650250444000333 